EXTENDED REPORT

\title{
Long term prognosis of children born to lupus patients
}

\author{
A Murashima, T Fukazawa, M Hirashima, Y Takasaki, M Oonishi, S Niiijima, Y Yamashiro, \\ A Yamataka, T Miyano, H Hashimoto
}

Ann Rheum Dis 2004;63:50-53. doi: 10.1136/ard.2002.001594

See end of article for
authors' affiliations
......................
Correspondence to:
Dr A Murashima,
Department of Maternal
Medicine, National Centre
for Child Health and
Development, 2-10-1
Okura Setagaya-ku,
Tokyo, 157-8535 Japan;
murasima-a@ncchd.go.jp

Accepted 12 May 2003

\begin{abstract}
Objective: To determine the long term prognosis of children of patients with systemic lupus erythematosus (SLE).

Methods: Children of patients with SLE were invited to attend our clinic for physical examination and laboratory tests. A total of 195 children (aged 4 months to 26 years; male $=82$, female $=113$ ) were examined in 1991, 1995, 1997, and 1998.

Results: Two cases were diagnosed as SLE at the first visit and were excluded from the second visit. A significantly higher percentage $(52 / 195(27 \%))$ of patients were positive for antinuclear antibodies (ANA) at a cut off serum dilution of 1/40 compared with controls $(4 / 57(7 \%))$. ANA were detected more frequently in female subjects than in men $(p<0.05)$. Forty four subjects were examined on more than two occasions. Nine of the 10 patients who were positive for ANA at the second visit were girls aged 4-8 years. The incidence of anti-DNA and antiphospholipid antibodies in children of patients with SLE was similar to that in the controls.

Conclusions: The finding that children, especially girls, born to maternal lupus patients had a high positive rate for ANA suggests that a genetic factor is involved in SLE pathogenesis. Longitudinal observation of these patients may provide important clinical information and clues to the pathogenesis of SLE.
\end{abstract}

S ystemic lupus erythematosus (SLE) is a chronic autoimmune inflammatory disease with heterogeneous clinical features, which primarily affects women in their childbearing years. The familial nature of SLE is well known, and thus patients with SLE may worry that their children will be affected. This study was therefore designed to acquire more information about the long term prognosis of children of patients with lupus.

\section{PATIENTS AND METHODS}

Letters were sent to children born to patients with SLE at Juntendo University Hospital in 1991, 1995, 1997 and 1998 informing them of this study. Informed consent was obtained from the children themselves or their parents. The children were interviewed about their medical condition, and each child was given a physical examination by a paediatrician or a doctor and laboratory tests were performed as described below. Control serum samples were obtained from other children admitted to our hospital for minor surgery.

Antinuclear antibodies (ANA) were assayed by an immunofluorescence technique using HEp-2 cells as the substrate. Anti-double stranded DNA (dsDNA) antibodies were measured by the Farr assay and enzyme linked immunosorbent assay (ELISA) with a commercially available kit (MESACUP-DNAII, Nagoya, Japan) using antihuman $\gamma$ chain monoclonal antibodies as secondary antibodies. Antisingle stranded DNA (ssDNA) antibodies were assayed by ELISA with the same kit (MESACUP-DNAII, Nagoya, Japan). Anticardiolipin antibodies (aCL) (IgG, IgM) were measured by a standardised ELISA. ${ }^{1}$ IgG antibodies against the cardiolipin and $\beta_{2}$-glycoprotein I (aCL/ $\left.\beta_{2} \mathrm{GPI}\right)$ complex were assessed by ELISA (aCL $/ \beta_{2}$ GPI EIA kit, Yamasa, Tokyo, Japan). Extractable antinuclear antigen (ENA) antibodies were detected by double immunodiffusion using rabbit thymus extract which was prepared as previously reported. ${ }^{2}$ Statistical analysis was performed with the $\chi^{2}$ test.

\section{RESULTS}

One hundred and ninety five children ( 82 male, 113 female), aged 4 months to 26 years were enrolled in the study. Of the
195 children, 56 were seen in 1991, 47 in 1995, 54 in 1997, and 38 in 1998. Forty nine children were siblings; none of them were twins. Two children were diagnosed with SLE at the first visit. One of them was a subject with neonatal lupus erythematosus who developed SLE at 6 months of age. The other developed SLE at 18 years of age. These two children were excluded from the following study because they had received treatment and were not suitable for a longitudinal study. Physical examination at the first visit showed that children born to patients with SLE had a greater tendency to have allergic diseases. ${ }^{3}$

Control serum samples were obtained from 57 children born to mothers without SLE.

\section{Antinuclear antibodies}

Table 1 shows the results for the ANA tests on the 195 children. The cut off point for ANA was defined as a serum dilution of $1 / 40$. Fifty two children of 195 patients with SLE $(27 \%)$ and only $4 / 57(7 \%)$ controls had ANA in their sera-a significant difference $(p<0.005)$. The presence of ANA did not show a sibling cluster. In 49 cases, ANA were stained in homogeneous and/or speckled patterns. The three exceptions had discrete-speckled, nuclear, and speckled patterns, respectively. When the prevalence of ANA in male and female children was compared (table 1), 41/113 (36\%) female children and 11/82 (13\%) male children were positive for ANA $(\mathrm{p}<0.001)$. When ANA were investigated according to age (fig 1), two of four sera from children younger than 6 months of age were positive for ANA, whereas all 40 sera from patients aged 6 months to 2 years were negative. The prevalence of ANA positivity was significantly higher in children older than 3 years than in those aged less than 3 years $(50 / 151(33 \%) v 2 / 44(5 \%) ; \mathrm{p}<0.001)$. For the children of patients with SLE about $50 \%$ of the ANA positive cases had

Abbreviations: ANA, antinuclear antibodies; CL, cardiolipin; ELISA, enzyme linked immunosorbent assay; ENA, extractable antinuclear antigen; GP, glycoprotein; SLE, systemic lupus erythematosus 
Table 1 Detection of ANA in children born to lupus patients and controls

\begin{tabular}{|c|c|c|c|}
\hline & No & Positive & $\%$ \\
\hline \multicolumn{4}{|c|}{ Children of patients with SLE } \\
\hline Male & 82 & 11 & $13^{*}$ \\
\hline Female & 113 & 41 & $36^{*}$ \\
\hline Total & 195 & 52 & $27^{* *}$ \\
\hline \multicolumn{4}{|l|}{ Controls } \\
\hline Male & 43 & 4 & 9 (NS) \\
\hline Female & 14 & 0 & 0 (NS) \\
\hline Total & 57 & 4 & $7^{* \star}$ \\
\hline
\end{tabular}

high titres of $>1 / 160$. In contrast, the four sera from controls that were ANA positive had low titres of $<1 / 160$ (data not shown).

Forty four children attended two, three, or four times (20, 11 , and 13 patients, respectively) for tests. Figure 2 shows the changes in ANA levels, excluding the children who were ANA positive or ANA negative in all investigations. One child younger than 6 months had ANA at the first investigation but became negative by the age of 4 years. This change distinguishes ANA transferred from the mother. Nine of 10 children who had become positive for ANA at the second investigation were aged 4-8 years. One girl became positive for ANA at age 14 years. Two boys and one girl became negative at the third investigation when they were 6,10 , and 8 years old. Excluding one infant under 6 months of age, eight children were ANA positive at the first investigation. One of them had become negative by the time of the third investigation at the age of 11 years. Seven children were continuously ANA positive. The other 25 children were ANA negative in all investigations.

\section{Anti-ENA antibodies}

Anti-ENA (Sm, UlRNP, Ro, La, Scl-70) antibodies were detected in 73 sera from children who attended the first and/ or second investigation. One of these serum samples was also positive for anti-UlRNP antibodies. This patient had few clinical symptoms, a characteristic typical of connective tissue diseases such as Raynaud's phenomenon.

\section{Anti-DNA antibodies}

Anti-DNA antibodies were measured by three methods in the sera of children who attended the first and/or second investigation (table 2). The Farr assay was negative, except in one child who was diagnosed with SLE. The results for the detection of anti-dsDNA antibodies by ELISA were the same as those of the Farr assay. Anti-ssDNA antibodies were positive in four of 77 sera $(5 \%)$. Of the 54 control sera, one had a low titre of anti-DNA antibodies by the Farr assay. Anti-dsDNA and anti-ssDNA measured by ELISA were negative in all control sera. However, the differences found between children born to patients with SLE and controls were not statistically significant.

\section{Anticardiolipin antibodies}

IgM aCL, IgG aCL, and $\beta_{2}$ GPI dependent aCL were measured in the sera of 73 children of patients with SLE and 54 controls (table 2). IgM aCL were positive in four sera from children of patients with SLE and in one control serum. IgG aCL were positive in one serum from each group (1.4\%, $1.9 \%$, respectively). All sera in each group were negative for anti$\mathrm{CL} / \beta_{2}$ GPI antibodies. There was no significant difference in the percentage of children of patients with SLE and controls who were aCL positive.

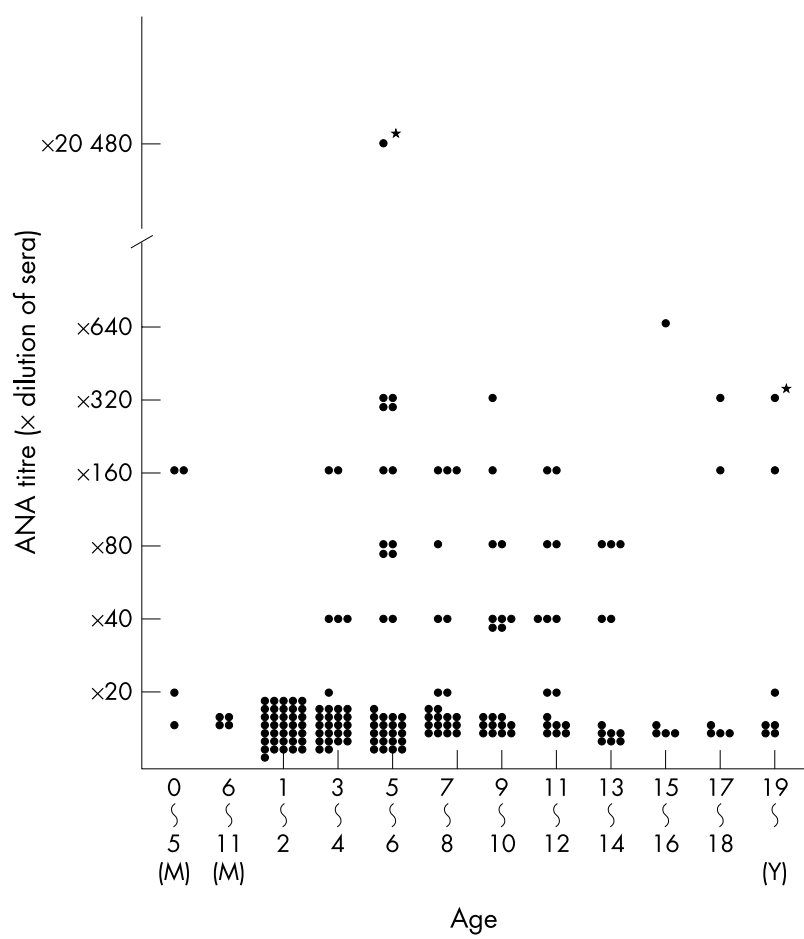

Figure 1 Presence of ANA in children born to lupus patients $(n=195)$. Except for two children younger than 6 months of age, all of the children with ANA were older than 3 years. Many cases were positive at a higher dilution. *Patient with SLE.

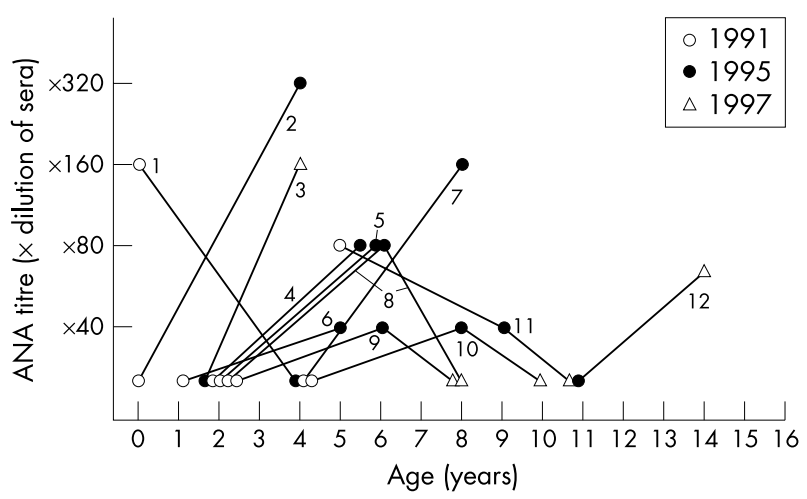

Figure 2 The change of ANA levels in the children who attended more than twice (excluding children who were ANA positive or ANA negative at all investigations). One infant younger than 6 months of age was ANA positive at the first investigation, but had become negative at the second (1). Nine children (one male, eight female) had become positive for ANA at the time of the second investigation when they were 4-8 years of age (2-10). Three of them (one boy (9), two girls $(8,10)$ ) had become negative again by the time of the third investigation. One girl became positive at 14 years old (12). One boy was positive at 5 years of age and had become negative by 11 years of age (11).

\section{DISCUSSION}

Studies using genetic epidemiology and molecular biology techniques have suggested that genetic factors may have an important role in the pathogenesis of SLE.

Many studies have shown that relatives of patients with SLE often have the same disorder or another autoimmune disease. ${ }^{45}$ When the serological abnormalities are investigated, the frequency of this link is more striking. ${ }^{6}$ An increase in ANA positivity was found in the sera of relatives of patients with SLE, including not only offspring but also siblings. That study which focused on the female first degree 
Table 2 Presence of autoantibodies including anti-DNA antibodies and antiphospholipid antibodies in children born to lupus patients and controls

\begin{tabular}{lll}
\hline & $\begin{array}{l}\text { Children with SLE } \\
\text { positive/total (\%) }\end{array}$ & $\begin{array}{l}\text { Controls positive/ } \\
\text { total (\%) }\end{array}$ \\
\hline Anti-dsDNA & & \\
By Farr assay & $1^{*} / 179(0.6)$ & $1 / 54(1.9)$ \\
By ELISA & $1^{* / 78(1.6)}$ & $0 / 54$ \\
Anti-sLDNA & $4^{*} / 78(5.1)$ & $0 / 54$ \\
Anti-CL(IgM) & $4 / 73(1.5)$ & $1 / 54(1.9)$ \\
Anti-CL(IgG) & $1 / 73(1.4)$ & $1 / 54(1.9)$ \\
Anti- $\beta_{2}$ GPI & $0 / 73$ & $0 / 54$ \\
\hline
\end{tabular}

ELISA, enzyme linked immunosorbent assay; CL, cardiolipin; GP, glycoprotein.

*Including one patient with SLE.

relatives detected ANA in $20 \%{ }^{7}$ To date, there have been no longitudinal studies which focused only on the children born to patients with SLE, although there have been a small number of reports of studies involving newborn babies. There also seem to be no prospective studies investigating the offspring of probands with SLE. We investigated the long term prognosis of a large number of children born to maternal lupus patients.

In this study, two of 56 children were diagnosed as SLE at the first visit, representing a prevalence of around $4 \%$. This is similar to the prevalence of mother-child cases reported elsewhere $e^{8}$ and clearly higher than the overall prevalence of SLE in Japan, which is reported to be $0.21 \% .{ }^{9}$ Interviews with, and physical examination of, the 56 children at the first survey indicated a high prevalence of atopic disorders compared with the controls, ${ }^{3}$ although this could not be investigated further as no paediatric allergist was available.

The presence of autoantibodies in the sera of newborn babies of mothers with SLE has been reported previously. ${ }^{1011}$ However, it is likely that these autoantibodies were transferred from the mother through the placenta. The serological characteristics of older children of mothers with SLE have not been reported so far. When ANA were investigated, the percentage of children who were ANA positive was significantly higher than that of the controls. Investigation according to age showed that all ANA positive children were aged over 3 years, when neonates under 6 months of age were excluded, because the IgG class of antibodies might be detected in the serum of infants owing to transplacental passage of maternal immunoglobulin. The rate of ANA positivity was also 2.5 times higher in female than in male children. This ratio is similar to those demonstrated in familial cases of SLE and SLE in childhood. ${ }^{12}$ This discrepancy between the sexes might be considered as due to hormonal, environmental, behavioural, or genetic effects. Hormonal effects must exclude oestrogen because serum oestrogen levels are the same for boys and girls. During childhood, there is no reason to suggest that differences in environment or behaviour lead to differences in ANA positivity between the sexes. Therefore, studies of the X and Y chromosomes such as imprinting and $X$ inactivation ${ }^{13}$ may provide information, but such studies have rarely been performed.

Some control sera in our study were also positive for ANA. It has been reported that healthy people seldom have ANA in their sera. The most recent multicentre study, ${ }^{14}$ however, showed that about $20 \%$ of healthy adults had ANA in their sera when a cut off dilution of $1 / 40$ was used. In the few reports published, the frequency of ANA in healthy children using HEp-2 cells as a substrate was between $0.4 \%^{15}$ and $9 \%^{16}$ at a dilution of $1 / 40$. Our study included age matched control sera from children admitted for minor surgery. Four out of 57 $(7 \%)$ serum samples were positive for ANA. It is unclear whether detection of ANA in the sera of controls has the same biological significance as its detection in children born to maternal lupus patients. There was a marked difference between the ANA positivity. Thus the maximum titre of ANA in the controls was $1 / 160$, whereas that in children born to maternal lupus patients was $1 / 640$, except for two children with SLE. This study has shown that the children born to lupus patients had not only a higher frequency of ANA but also a higher titre of ANA than controls.

The changes in ANA titres according to age were studied. Interestingly, many patients became positive for ANA between 4 and 8 years of age, but some of them reverted to negative at over 8 years. We did not detect any clinical manifestations related to SLE in cases of seroconversion. The causes of this seroconversion are not clear. To answer this question, further studies, including examination of the age related immunological function and apoptosis of autoreactive lymphocytes or ANA producing lymphocytes, are needed. Recent studies have reported relationships between microchimerism and the autoimmune phenomenon. ${ }^{17}$ Maternal cells can persist in the peripheral blood of children for a long time and those may be involved in the pathogenesis of juvenile onset rheumatological diseases. ${ }^{18}$ This theory also provides a plausible explanation for both the seroconversion and reversion phenomena.

Anti-DNA antibodies were measured by three methods. Anti-dsDNA antibodies measured by the Farr assay and ELISA were negative in all children except for one with SLE. Only $5 \%$ of patients were positive for anti-ssDNA antibodies. These results were not noteworthy because the detection of anti-ssDNA antibodies in the sera is less specific than that of anti-dsDNA antibodies.

Anti-UIRNP was detected in one serum sample only. This antibody is specific to certain diseases or symptoms. As this subject was asymptomatic, a longer follow up is required to determine the meaning of this result. The mother of this child tested positive for anti-proliferating cell nuclear antigen antibodies. This result supports a previous study which showed that autoantibodies in family members are not necessarily directed against the same nuclear antigens. ${ }^{19}$

IgG aCL and IgM aCL were positive in $1.4 \%$ and $5.5 \%$ of children born to lupus patients, respectively. They were also detected in the sera of controls. There were no differences in the prevalence of these antibodies between the children born to lupus patients and controls. The cases testing positive for IgG aCL and IgM aCL in both groups were not associated with acute or recent infection. No anti-CL/2GPI antibodies were detected in either children born to patients with SLE or controls. The presence of IgG aCL in some children was unlikely to be due to transfer from the mother because the patients were over 6 months of age. These patients had no clinical symptoms associated with antiphospholipid syndromes.

This study has shown that many children born to patients with SLE, especially children over 3 years of age had ANA in their sera. Furthermore, follow up of these patients for eight years showed that the presence of ANA varies from age to age. These results may be useful when informing parents about the long term impact of maternal lupus on their children. Further studies are necessary to know the reason why ANA are frequently found in children born to lupus patients. The answer to this may provide a clue to clarify the pathogenesis of SLE.

\footnotetext{
Authors' affiliations

A Murashima, Department of Maternal Medicine, National Centre for Child Health and Development, Tokyo, Japan

A Murashima, T Fukazawa, M Hirashima, Y Takasaki, H Hashimoto, Department of Internal Medicine and Rheumatology, Juntendo University School of Medicine, Tokyo, Japan
} 
M Oonishi, S Niijima, Y Yamashiro, Department of Paediatrics, Juntendo University School of Medicine, Tokyo, Japan

A Yamataka, T Miyano, Department of Paediatric Surgery, Juntendo University School of Medicine, Tokyo, Japan

\section{REFERENCES}

1 Harris EN, Ghavari AE, Hughes GRV. The anti-cardiolipin assay, In: Harris EN, Exner T, Hughes GRV, Asherson RA, eds. Phospholipid-binding antibodies. Boca Raton, FL: CRC Press, 1991:175-87.

2 Takasaki Y, Fishwild D, Tan EM. Characterization of proliferating cell nuclear antigen recognized by autoantibodies in lupus sera. J Exp Med 1984:159:981-92.

3 Sasai K, Furukawa S, Hashimoto H, Yabuta K. Increased levels of serum lgE in children of mothers with systemic lupus erythematosus. Allergy 1995;50:370-3.

4 Brunjes S, Zike K, Julian R. Familial systemic lupus erythematosus. Am J Med 1961;30:529-36.

5 Lawrence JS, Martins CL, Drake GL. A family survey of lupus erythematosus 1 . Heritability. J Rheumatol 1987; 14:913-21.

6 Arnett FC. The genetics of human lupus. In: Wallace DJ, Hahn BW, eds. Dubois' lupus erythematosus. 5th ed. Baltimore: Williams \& Wilkins, 1997:101-3.

7 Leonhardt T. Family studies in SLE. Clin Exp Immunol 1967;2:743-59.

8 Buckman KJ, Moore SK, Ebbin AJ, Cox MB, Dubois EL. Familial systemic lupus erythematosus. Arch Intern Med 1978:138:1674-16.

9 Nakae K, Furasa WF, Kasukawa R, et al. A nationwide epidemiological survey on diffuse collagen diseases; estimation of prevalence rate in Japan.
In: Kasukawa R. Missouri GL, eds. Mixed connective tissue disease and antinuclear antibodies. Amsterdam: Elsevier, 1987:9-20.

10 EL-Roeiy A, Gleicher N, Isenberg D, Kennedy RC, Shoenfeld Y. A common anti-DNA idiotype and other autoantibodies in sera of offspring of mothers with SLE. Clin Exp Immunol 1987:68:528-34.

11 Zurgil N, Bakimer R, Tincani A, Faden D, Cohen J, Lorber M, et al. Detection of anti-phospholipid and anti-DNA antibodies and their idiotypes in newborns of mothers with anti-phospholipid syndrome and SLE. Lupus 1993;2:233-7.

12 Takei S, Maeno N, Shigemori M, Imanaka H, Hokonohara M, Miyata K, et al. Clinical features of Japanese children and adolescents with systemic lupus erythematosus: results of 1980-1994 survey. Acta Paediatr Jpn 1997;39:250-6.

13 Tan EM, Feltkamp TEW, Smolen JS, Butcher B, Dawkins R, Takasaki Y, et al. Range of antinuclear antibodies in "healthy" individuals. Arthritis Rheum 1997;40:1601-111.

14 Stewart JJ. The female $X$-inactivation mosaic in systemic lupus erythematosus. Immunol Today 1998; 19:352-7.

15 Arroyave CM, Giambrone MJ, Rich KC, Walaszek M. The frequency of antinuclear antibody in children by use of mouse kidney (MK) and human epithelial cells (HEp-2) as substrates. J Allergy Clin Immunol 1988;82:741-74.

16 Osborn TG, Patel NJ, Moore TL, Zuckner J. Use of the HEp-2 cell substrate in the detection of antinuclear antibodies in juvenile rheumatoid arthritis. Arthritis Rheum 1984;27:1286-19.

17 Nelson Jl. Microchimerism and human autoimmune diseases. Lupus 2002;11:651-4.

18 Artleft CM, Miller FW, Rider LG. Persistent maternally derived peripheral microchimerism is associated with the juvenile idiopathic inflammatory myopathies. Rheumatology (Oxford) $2001 ; 40: 1279-84$.

\section{$\mathrm{ECHO}$}

\section{Inflammatory cytokines and rheumatoid corneal ulceration}

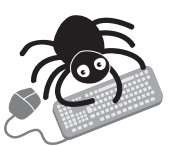

Please visit the Annals of the Rheumatic Diseases website [www. annrheumdis. com] for a link to the full text of this article.
$T$ he ocular complications of rheumatoid arthritis include aqueous tear deficiency, scleritis, and central or paracentral corneal ulceration or perforation. The corneal ulcers are said to be non-inflammatory but ultrastructural changes have been found in the corneal stroma surrounding ulcers. Electron-dense deposits, thought possibly to indicate aggregated collagen, have been seen in the extracellular stromal matrix. On light microscopy, however, there are few inflammatory cells. Researchers in Germany have demonstrated increased gene expression for TNF- $\alpha$ and IL- 6 in keratocytes around corneal ulcers or perforations in patients with rheumatoid arthritis.

They examined corneal samples from seven patients with rheumatoid arthritis and ulceration or perforation, using systematic non-radioactive in situ hybridisation with gene probes for TNF- $\alpha$ and IL-6 labelled with digoxigenin. Corneal samples from two patients with keratoconus were used as controls. High levels of IL-6 mRNA were demonstrated in keratocytes of all seven patients and of TNF- $\alpha$ mRNA in five of the seven. The two control samples gave negative results. TNF- $\alpha$-positive keratocytes were seen mostly around the corneal epithelium whereas IL-6-positive keratocytes tended to be deeper in the stroma. The two patients with low TNF- $\alpha$ gene expression both had acute infections, one with herpes zoster and one with Staphylococcus species. The authors comment that neither of these patients had clear rheumatic symptoms confirmed.

TNF- $\alpha$ and IL- 6 are both expressed in keratocytes around the corneal ulcers or perforations of patients with rheumatoid arthritis. It is suggested that these cytokines may modify metalloproteinase production, causing lysis of collagen. Inflammatory cytokine production may be a cause or a consequence of corneal ulceration or perforation in patients with rheumatoid arthritis.

A British Journal of Ophthalmology 2003;87:548-550. 